\title{
A SIMPLE MATHEMATICAL MODEL FOR HIGH TEMPERATURE SUPERCONDUCTIVITY
}

\author{
Masao Nagasawa
}

\begin{abstract}
There exists no established theory for high temperature superconductivity, since Müller and Bednorz's discovery in 1986, although some partial theories have been proposed. We will give a consistent mathematical theory of high temperature superconductivity based on a theory of stochastic processes of Schrödinger and Nagasawa.
\end{abstract}

\section{Introduction}

Kamerling Onnes discovered in 1911 an interesting phenomenon that the electric resistance of $\mathrm{Hg}$ suddenly disappears at the absolute temperature $4.2 \mathrm{~K}$ and the electric current flows continuously undisturbed. This phenomenon is called "superconductivity". After his discovery, metals with higher transition temperatures were looked for, but the highest transition temperature was about $10 \mathrm{~K}$.

A brake-through was made by Müller and Bednorz in 1986. They discovered that a ceramic $\mathrm{La}_{2} \mathrm{CuO}_{4}$ (precisely speaking, some percent of $\mathrm{La}$ are replaced by $\mathrm{Sr}: \mathrm{La}_{2-x} \mathrm{Sr}_{x} \mathrm{CuO}_{4}$ ) shows the superconductivity at $40 \mathrm{~K}$. This phenomenon was called "high temperature superconductivity". "High temperature" means that the transition temperature $40 \mathrm{~K}$ is remarkably high compared to the transition temperature $4.2 \mathrm{~K}$ of $\mathrm{Hg}$. However, $40 \mathrm{~K}$ is still an extremely low temperature compared to the normal temperature $300 \mathrm{~K}$. Up to now the highest transition temperature observed under normal pressure is $95 \mathrm{~K}$ of $\mathrm{YBa}_{2} \mathrm{Cu}_{3} \mathrm{O}_{7-y}$. (Superconductors with higher transition temperatures have been discovered under high pressure.)

The ceramic of Müller and Bednorz consists of layers of copper oxide, which will be called "yellow layers", and layers without copper oxide, which will be called "white layers", as illustrated:

2010 Mathematics Subject Classification. 60G99.

Key words and phrases. mathematical model for high temperature superconductivity, BoseEinstein condensation.

Received May 15, 2013; revised September 26, 2013. 


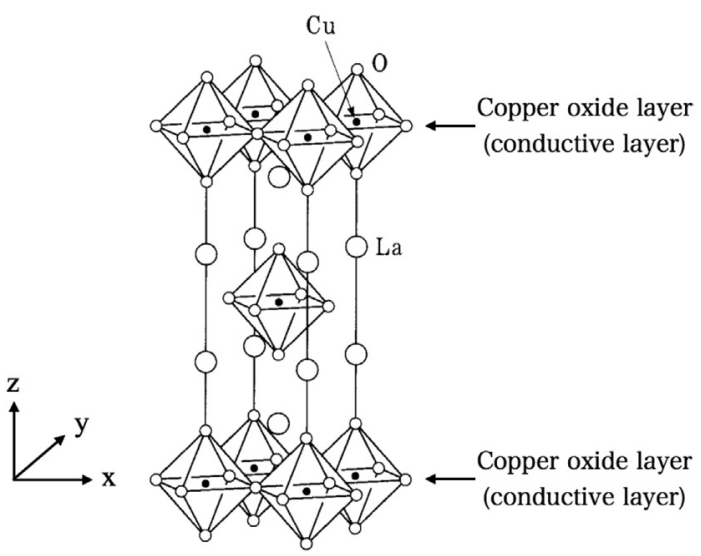

Movable particles (electrons or positive holes of electrons) stay in a yellow layer, which will be defined as $\mathbf{R}^{2} \times[0, \ell], \ell>0$, and don't move across white layers.

There exists, however, no established theory for high temperature superconductivity, as far as my knowledge. We will, therefore, give a theory of high temperature superconductivity based on a simple mathematical model.

\section{Mathematical model of superconductivity}

Electrons are fermions, and don't obey Bose-Einstein statistics. Accordingly, one can't immediately apply the theory of Bose-Einstein condensation in discussing the transition to superconductivity of electron gas. We need, therefore, additional ideas to solve the problem.

In an accepted theory of Bardeen-Cooper-Schrieffer (1957) for low temperature superconductivity, two electrons are coupled and regarded as a boson, and the theory of Bose-Einstein condensation was applied to these bosons. This is an interesting idea, and was successful in discussing low temperature superconductors, but has not given comprehensive understanding of high temperature superconductivity so far, because it is a difficult task to fit the crystal (layer) structure of high-temperatue superconductors to BCS theory.

We will therefore put BSC aside, and take a different way to discuss high temperature superconductivity. It is based on a simple mathematical idea:

The layer structure of high-temperature superconductors might play a decisive role for the occurrence of superconductivity. Based on this observation we will look at "the components of the motions of movable particles orthogonal to the copper-oxide-layer" and regard the set of orthogonal components of the motions of particles as "a set of one-dimensional bose particles".

Let us denote a yellow layer as $\mathbf{R}^{2} \times[0, \ell]$. We regard the direction orthogonal to the $x y$-plane $\mathbf{R}^{2}$ as the $z$-axis. If we look at the crystal structure 
of the high-temperatur superconductor $\mathrm{La}_{2} \mathrm{CuO}_{4}$ in this coordinate system, we find that the $z$-direction and $x, y$-direction play completely different roles. In fact, there are upper and lower boundaries in the $z$-direction, while there is no such restriction in the $x, y$-direction. Therefore, it is plausible to assume that the superconductive current occurs on the $x y$-plane and the component along the $z$-axis concerns the transition to superconductivity.

This can be pictured as follows: Imagine many needles are standing on the $x y$-plane (a forest of needles), where lengths of needles are various and changing in time. ("Needle" is just to help making an image, and has no further meaning.) On each needlepoint sits a movable particle. If we watch arbitrarily chosen two needles in the forest, they are allowed to have the same length and to make the same up-down motion at the same time, because the $x y$-components of our particles may be different. Therefore, even though movable particles are fermions, their $z$-components may be treated as bosons.

We now collect all needles and put them on the $z$-axis. (Needles' standing points on the $x y$-plane no longer play any role.) We denote by $z(t)$ the value of a needlepoint on the $z$-axis, and look at the up-down motion of the needlepoint $z(t)$. What we observe is, therefore, the set of $\{$ all "needlepoints" $\}$, that is, the set $\boldsymbol{Z}=\left\{z\right.$-components of all movable particles in a yellow layer $\left.\mathbf{R}^{2} \times[0, \ell]\right\}$. If the lengths of needles may become the same and make the same up-down motion at the same time, the set of $\{$ all "needlepoints" $\}$ is a system of one-dimensional bose particles.

The model considered above can be formulated with Schrödinger functions. We assume that the Schrödinger function $\psi_{t}\left((x, y, z)_{1},(x, y, z)_{2},(x, y, z)_{3}, \ldots\right)$ which describes the motion of movable particles (electrons or positive holes of electrons) in a yellow layer $\mathbf{R}^{2} \times[0, \ell]$ can be decomposed as

$$
\begin{aligned}
& \psi_{t}\left((x, y, z)_{1},(x, y, z)_{2},(x, y, z)_{3}, \ldots\right) \\
& \quad=\psi_{t}\left((x, y)_{1},(x, y)_{2},(x, y)_{3}, \ldots\right) \times \psi_{t}\left(z_{1}, z_{2}, z_{3}, \ldots\right),
\end{aligned}
$$

and

$$
\psi_{t}\left((x, y)_{1},(x, y)_{2},(x, y)_{3}, \ldots\right) \text { is antisymmetric, }
$$

while

$$
\psi_{t}\left(z_{1}, z_{2}, z_{3}, \ldots\right) \text { is symmetric. }
$$

Then, under this assumption of our mathematical model,

$\psi_{t}\left(z_{1}, z_{2}, z_{3}, \ldots\right)$ is the Schrödinger function of the z-components of the motion of movables particles in a yellow layer $\mathbf{R}^{2} \times[0, \ell]$, and it describes the motion of a set of bose particles on the z-axis.

Accordingly, we can apply the Bose-Einstein statistics to our onedimensional boson gas on the $z$-axis, and discuss the Bose-Einstein condensation of this boson gas on the $z$-axis.

Therefore, under the assumption above, we can conclude: 
The set $\boldsymbol{Z}=\{z$-components of all movable particles (electrons or positive holes) in a yellow layer $\left.\mathbf{R}^{2} \times[0, \ell]\right\}$ is a boson gas and makes the Bose-Einstein condensation.

With this fact in mind, we now look at the motion of movable particles in a yellow layer. Then the random motion orthogonal to the xy-plane falls down into the ground state at the time that the Bose-Einstein condensation occurs. What remains after that is therefore the drift-motion on the xy-plane only. This driftmotion which remains on the xy-plane is actually "the permanent electric current" of our superconductor.

For the statement above see Proposition 1 in the following section.

Remark. The random motion of the $z$-component works as a disturbance to the electric current on the $x y$-plane, i.e., as electric resistance. This implies that if the $z$-component of the motion falls down into the ground state, it is the minimal unavoidable disturbance to the current on the $x y$-plane. There exists therefore no better state of existence for the electric current. This is the so-called superconductivity.

\section{High temperature superconductivity}

We consider the ceramic $\mathrm{La}_{2} \mathrm{CuO}_{4}$ of Müller and Bednorz. A movable particle (electron or positive hole of an electron) stays in a yellow layer $\mathbf{R}^{2} \times[0, \ell]$, and doesn't move across white layers, where $\ell$ may be regarded as a thickness of a yellow layer, which should be determined by the crystal structure of each high temperature superconductor. (To determine the physical quantity $\ell$ of each superconductor is entrusted as a problem for physicists.)

Because of the crystal structure, the motion of every movable particle along the $z$-axis is confined, but the motion on the $x y$-plane has no such restriction. We will show that the $z$-component of the motion will fall down into the ground state and work as the minimal electric resistance, while the $x y$-component of the motion will be a (permanent) electric current. Thus our superconductor's crystal structure along the $z$-axis and $x y$-plane play different roles.

We will follow the observation above, and analyze first of all the $z$ component of the motion of a movable particle, which is a one-dimensional motion on the $z$-axis confined in an interval $[0, \ell], \ell>0$.

If we confine the motion of a particle on the $z$-axis in an interval $[0, \ell]$, then the eigenvalue problem of the motion is

$$
\frac{d^{2} \Phi}{d z^{2}}+\frac{2 m}{\hbar^{2}}(\lambda-V(z)) \Phi=0
$$

where the potential function $V(z)$ is

$$
\begin{gathered}
V(z)=0, \quad z \in[0, \ell], \\
V(z)=\infty, \quad z \notin[0, \ell] .
\end{gathered}
$$


In this case, eigenfunctions are

$$
\Phi=\sqrt{\frac{2}{\ell}} \sin \frac{n \pi z}{\ell}, \quad n=1,2,3, \ldots,
$$

and eigenvalues are

$$
\lambda=\frac{\hbar^{2}}{2 m} \pi^{2} \frac{n^{2}}{\ell^{2}}, \quad n=1,2,3, \ldots
$$

These are possible energies of the random motion of $z$-components of movable particles in a yellow layer $\mathbf{R}^{2} \times[0, \ell]$.

We now consider a set

$\boldsymbol{Z}=\left\{z\right.$-components of all movable particles in a yellow layer $\left.\mathbf{R}^{2} \times[0, \ell]\right\}$.

As explained in our mathematical model of superconductivity, we can assume that $\boldsymbol{Z}$ is a set of independent bose particles.

Therefore, the mean number $\left\langle n_{\varepsilon}\right\rangle$ of particles with the energy $\varepsilon$ is given by the Bose-Einstein distribution

$$
\left\langle n_{\varepsilon}\right\rangle=\frac{1}{\exp \left((\varepsilon-\mu) / k_{B} T\right)-1},
$$

where $\mu$ is the chemical potential, $k_{B}$ is the Boltzmann constant and $T$ is the temperature (cf. Einstein $(1924,1925)$ ).

Then the mean number $N_{\mathrm{ex}}$ of particles in excited states is given by

$$
N_{\mathrm{ex}}=\sum_{\varepsilon}\left\langle n_{\varepsilon}\right\rangle
$$

In equation (2) we set $\varepsilon=\frac{\hbar^{2}}{2 m} \pi^{2} \frac{n^{2}}{\ell^{2}}$ as obtained in equation (1), and $\mu=0$. Then the mean number $N_{\mathrm{ex}}$ of particles in excited states is given by

$$
N_{\text {ex }}=\sum_{n=1}^{\infty} \frac{1}{\exp \left(\frac{\hbar^{2}}{2 m} \pi^{2} \frac{n^{2}}{\ell^{2}} / k_{B} T\right)-1} .
$$

We denote $a=\frac{\hbar^{2}}{2 m} \pi^{2} \frac{1}{\ell^{2}} / k_{B} T \ll 1$ and replace summation by integration

$$
\begin{aligned}
N_{\mathrm{ex}} & =\sum_{n=1}^{\infty} \frac{1}{\exp \left(a n^{2}\right)-1} \\
& =\int_{1}^{\infty} \frac{1}{\exp \left(a x^{2}\right)-1} d x
\end{aligned}
$$


and set $y=\exp \left(a x^{2}\right)$

$$
=\frac{1}{2 \sqrt{a}} \int_{e^{a}}^{\infty} \frac{1}{y(y-1)} \frac{1}{\sqrt{\log y}} d y .
$$

Then, since the only $y$ near by $e^{a}$ contributes mainly to the integral, we have

$$
\simeq \frac{1}{2 a} \int_{e^{a}}^{\infty} \frac{1}{y(y-1)} d y,
$$

where the indefinite integral of $\frac{1}{y(y-1)}=-\frac{1}{y}+\frac{1}{y-1}$ is $\log \left|\frac{y-1}{y}\right|$,

$$
\begin{aligned}
& =\frac{1}{2 a}\left[\log \left|\frac{y-1}{y}\right|\right]_{e^{a}}^{\infty} \\
& =\frac{1}{2 a} \log \left|\frac{e^{a}}{e^{a}-1}\right|,
\end{aligned}
$$

where $a=\frac{\hbar^{2}}{2 m} \pi^{2} \frac{1}{\ell^{2}} / k_{B} T \ll 1$ and

$$
\simeq \frac{1}{2 a} \log \frac{1}{a}
$$

Therefore,

$$
N_{\mathrm{ex}} \simeq \frac{1}{2} \frac{k_{B} T}{\frac{\hbar^{2}}{2 m} \pi^{2} \frac{1}{\ell^{2}}} \log \frac{k_{B} T}{\frac{\hbar^{2}}{2 m} \pi^{2} \frac{1}{\ell^{2}}} .
$$

Now let $N$ be the number of particles per unit volume. If the temperature $T$ is high enough and $N_{\text {ex }}>N$, then all particles are in excited states. We now lower the temperature $T$. Then $N_{\text {ex }}$ decreases and will be $N_{\text {ex }}<N$.

We denote the critical temperature by $T_{c}$ at which $N_{\mathrm{ex}}=N$. Then

$$
k_{B} T_{c} \simeq \frac{\hbar^{2}}{2 m} \pi^{2} \frac{1}{\ell^{2}} \frac{2 N}{\log 2 N},
$$

that is, the critical temperature $T_{c}$ is determined by a structure constant $\ell^{2}$ of our crystal and the number $N$ of particles in our electron gas.

According to Einstein $(1924,1925)$, the gas of particles is saturated at the temperature $T=T_{c}$. Since the number $N_{\text {ex }}$ is less than $N$ at $T<T_{c}$, the number

$$
N_{0}=N-N_{\text {ex }}
$$

of particles must be condensed into the state of the lowest energy. This is the socalled Bose-Einstain condensation. In other words, the motion along the $z$-axis falls down into the lowest energy state. 
A SIMPLE MATHEMATICAL MODEL FOR HIGH TEMPERATURE SUPERCONDUCTIVITY 253

Definition 1. We will call the critical temperature $T_{c}$ given in equation (4) "the transition temperature for high temperature superconductivity".

The transition temperature $T_{c}$ of a high temperature superconductor depends on $\frac{1}{\ell^{2}}$, that is, on its crystal structure and the number $N$ of moveable particles per unit volume.

Remark. About the Bose-Einstain condensation in lower dimensions we refer to e.g. Görlitz et al. (2001), section 2.6 of Pethick-Smith (2002).

If the temperature goes down, then the kinetic energies of particles decrease, but in our model the kinetic energy of the motion along the $z$-axis goes down to the lowest energy state at the transition temperature $T_{c}$, that is, the spatial symmetry of the motion of particles remarkably breaks down.

We now consider the motion of movable particles in a yellow layer $\mathbf{R}^{2} \times[0, \ell]$ at the temperature $T \leq T_{c}$.

Let the motion of particles be determined by a complex evolution function (Schrödinger function)

$$
\psi_{t}(x, y, z)=e^{R(t, x, y, z)+i S(t, x, y, z)}
$$

or, what is the same, by a pair of evolution functions

$$
\phi_{t}(x, y, z)=e^{R(t, x, y, z)+S(t, x, y, z)}, \quad \hat{\phi}_{t}(x, y, z)=e^{R(t, x, y, z)-S(t, x, y, z)} .
$$

To be precise, $\psi_{t}(x, y, z)$ or a pair $\left\{\phi_{t}(x, y, z), \hat{\phi}_{t}(x, y, z)\right\}$ determines the motion of the set of movable particles (electron gas) in a yellow layer $\mathbf{R}^{2} \times[0, \ell]$ as follows:

If $\psi_{t}(x, y, z)$ or a pair $\left\{\phi_{t}(x, y, z), \hat{\phi}_{t}(x, y, z)\right\}$ is given, then a pair of functions $R(t, x, y, z)$ and $S(t, x, y, z)$ determine the so-called "evolution-drift"

$$
\boldsymbol{a}(t, x, y, z)=\sigma^{2} \nabla(R(t, x, y, z)+S(t, x, y, z)),
$$

and "time-reversed evolution-drift"

$$
\hat{\boldsymbol{a}}(t, x, y, z)=\sigma^{2} \nabla(R(t, x, y, z)-S(t, x, y, z)),
$$

with $\sigma^{2}=\frac{\hbar}{m}$, where they are defined by

$$
\boldsymbol{a}(t, x, y, z)=\sigma^{2} \nabla \log \phi_{t}(x, y, z) \quad \text { and } \quad \hat{\boldsymbol{a}}(t, x, y, z)=\sigma^{2} \nabla \log \hat{\phi}_{t}(x, y, z) .
$$

The evolution-drift $\boldsymbol{a}(t, x, y, z)$ is an extension of the notion of velocity in classical mechanics to the random motion of particles. Therefore, paths of a particle $X_{t}=\left(x_{t}, y_{t}, z_{t}\right)$ obey a stochastic differential equation

$$
X_{t}=X_{a}+\sigma B_{t-a}+\int_{a}^{t} \boldsymbol{a}\left(s, X_{s}\right) d s
$$

where $B_{t}=\left(B_{t}^{1}, B_{t}^{2}, B_{t}^{3}\right)$ is a three dimensional Brownian motion. 
For details of the dynamic theory of systems of random particles, cf., e.g. Nagasawa (1993, 2000, 2002, 2007, 2012*), or Appendix of Nagasawa (2012) for a short explanation.

Remark. The "evolution-drift" is first defined for the motion of a single particle. However, since a Schrödinger function describes also a system of particles (cf. Nagasawa $(1990,2012 *)$ ), the evolution-drift can be regarded as "the evolution-drift vector of a system of particles".

We now assume $T \leq T_{c}$ and analyze the motion of movable particles in a yellow layer $\mathbf{R}^{2} \times[0, \ell]$ for some details.

Because of the Bose-Einstein condensation, the motion along the $z$-axis is in the lowest energy state

$$
\Phi_{0}(z)=\sqrt{\frac{2}{\ell}} \sin \frac{\pi z}{\ell} .
$$

In this case the $z$-component of the evolution-drift is given by

$$
\sigma^{2} \frac{\partial \log \Phi_{0}(z)}{\partial z}=\sigma^{2} \frac{\pi}{\ell} \cot \frac{\pi z}{\ell} .
$$

Therefore, the motion along the $z$-axis is strongly repelled at the boundary points $z=0$ and $\ell$, and attracted to $z=\frac{\ell}{2}$ by the evolution-drift $\sigma^{2} \frac{\pi}{\ell} \cot \frac{\pi z}{\ell}$, and hence randomly oscillates at $z=\frac{\ell}{2}$.
Moreover,

$$
\begin{aligned}
& \frac{\partial R(t, x, y, z)}{\partial z}+\frac{\partial S(t, x, y, z)}{\partial z}=\frac{\pi}{\ell} \cot \frac{\pi z}{\ell} \\
& \frac{\partial R(t, x, y, z)}{\partial z}-\frac{\partial S(t, x, y, z)}{\partial z}=-\frac{\pi}{\ell} \cot \frac{\pi z}{\ell}
\end{aligned}
$$

must hold. We have, therefore,

$$
\frac{\partial R(t, x, y, z)}{\partial z}=0, \quad \frac{\partial S(t, x, y, z)}{\partial z}=\frac{\pi}{\ell} \cot \frac{\pi z}{\ell} .
$$

Thus we have shown:

Lemma 1. The evolution-drift vector $\boldsymbol{a}(t, x, y, z)$ of movable particles (electrons or positive holes) in a yellow layer is given by

(9) $\left(\sigma^{2} \frac{\partial(R(t, x, y)+S(t, x, y))}{\partial x}, \sigma^{2} \frac{\partial(R(t, x, y)+S(t, x, y))}{\partial y}, \sigma^{2} \frac{\pi}{\ell} \cot \frac{\pi z}{\ell}\right)$, 
where $\sigma^{2}=\frac{\hbar}{m} . \quad$ If

$$
\psi(t, x, y, z)=e^{i(E / \hbar) t} e^{R(x, y)+i(S(x, y)+\log (\sqrt{2 / \ell} \sin (\pi z / \ell)))},
$$

then on the xy-plane there exists a flow

$$
\boldsymbol{a}(x, y)=\left(\sigma^{2} \frac{\partial(R(x, y)+S(x, y))}{\partial x}, \sigma^{2} \frac{\partial(R(x, y)+S(x, y))}{\partial y}\right)
$$

of movable particles (electrons or positive holes). The z-component $\sigma^{2} \frac{\pi}{\ell} \cot \frac{\pi z}{\ell}$ of the drift vector in equation (9) no longer disturbs the flow a $(x, y)$ in equation (10), and hence the flow $\boldsymbol{a}(x, y)$ is a permanent electric current.

Lemma 1 implies, therefore,

Proposition 1. A permanent electric current a $(x, y)$ in equation (10) occurs at the transition temperature $T_{c}$ given by equation (4) (see equation (5) for the motion of particles), because the z-components of movable particles (electrons or positive holes) in a yellow layer $\mathbf{R}^{2} \times[0, \ell]$ fall down into the lowest energy state at $T_{c}$, and no longer disturb the electric current $\boldsymbol{a}(x, y)$.

Remark. According to the general theory of random motion, the kinetic energy of a particle is given by

$$
K(t, x, y, z)=\frac{1}{2 m} \hbar^{2}\left((\nabla R(t, x, y, z))^{2}+(\nabla S(t, x, y, z))^{2}\right) .
$$

Therefore, if the kinetic energy is constant and the $z$-component of the evolutiondrift vector becomes larger, then the $x y$-component decreases naturally, i.e., the $z$-component of evolution-drift vector works as electric resistance to the electric current $\boldsymbol{a}(x, y)$ on the $x y$-plane given in equation (10).

This is the reason why we observed the $z$-component of the motion of particles and its Bose-Einstein condensation first of all.

One of characteristic phenomena of superconductors is the so-called Meissner-Ochsenfeld effect, that is, if a magnetic field is weak, it can't invade into superconductors. This effect can be explained in our model as follows. Suppose a magnetic field invades into a superconductor. Then new momentum is induced to the motion along the $z$-axis. Consequently, the motion stays no longer in the lowest energy state. This means that the superconductivity is destroyed by the magnetic field. Therefore, in order to keep being a superconductor, any invasion of magnetic fields must be prevented.

If a magnetic field in the enviroment becomes stronger, it will then invade gradually from the surface of the superconductor and the superconductivity will be destroyed. 


\section{Low temperature superconductivity}

Our mathematical model of superconductivity can be applied also to the low temperature superconductivity, i.e., the case with no white layers. In this case we may choose the $z$-axis arbitrarily. We assume Hooke's potential for the motion along the $z$-axis, so that the motion makes random harmonic oscillation. This is a model hypothesis.

We set $\mu=\frac{1}{2} h v$ in equation (1). Since the energy of the one-dimensional harmonic oscillation is $\varepsilon=\left(n+\frac{1}{2}\right) h v$, the mean number of particles in excited states is

$$
N_{\text {ex }}=\sum_{n=1}^{\infty} \frac{1}{\exp \left(n h v / k_{B} T\right)-1} .
$$

We set $a=h v / k_{B} T$ in equation (11) and replace the summation by integration

$$
\begin{aligned}
& =\sum_{n=1}^{\infty} \frac{1}{\exp (a n)-1} \\
& =\int_{1}^{\infty} \frac{1}{\exp (a x)-1} d x .
\end{aligned}
$$

By changing variable as $\exp (a x)=y$, we have

$$
=\frac{1}{a} \int_{e^{a}}^{\infty} \frac{1}{y(y-1)} d y
$$

where the indefinite integral of $\frac{1}{y(y-1)}$ is $\log \left|\frac{y-1}{y}\right|$,

$$
\begin{aligned}
& =\frac{1}{a}\left[\log \left|\frac{y-1}{y}\right|\right]_{e^{a}}^{\infty} \\
& =\frac{1}{a} \log \left|\frac{e^{a}}{e^{a}-1}\right|
\end{aligned}
$$

where $a=h v / k_{B} T \ll 1$ and

$$
\simeq \frac{1}{a} \log \frac{1}{a}
$$

Thus we have

$$
N_{\mathrm{ex}} \simeq \frac{k_{B} T}{h v} \log \frac{k_{B} T}{h v} .
$$

Let $N$ be the number of particles per unit volume. If the temperature $T$ is high enough and $N_{\mathrm{ex}}>N$, then all particles are in excited states. We now lower the temperature $T$. Then $N_{\text {ex }}$ decreases and will be $N_{\text {ex }}<N$. 
We denote the critical temperature by $T_{c}$ at which $N_{\mathrm{ex}}=N$. Then

$$
k_{B} T_{c} \simeq h v \frac{N}{\log N} .
$$

Therefore, the critical temperature $T_{c}$ is determined by a structure constant $v$ of our superconductor and the number $N$ of movable particles.

According to Einstein $(1924,1925)$, the gas of particles is saturated at the temperature $T=T_{c}$. Since the mean number $N_{\text {ex }}$ is less than $N$ at $T<T_{c}$, the number

$$
N_{0}=N-N_{\mathrm{ex}}
$$

of particles must be condensed into the state of the lowest energy, that is, the Bose-Einstain condensation occurs. In other words, the motion along the $z$-axis falls down into the lowest energy state.

Definition 2. The critical temperature $T_{c}$ given in equation (13) is "the transition temperature for low temperature superconductivity".

The motion along the $z$-axis is in the lowest energy state, and the $z$ component of the evolution-drift is $-\sigma^{2} h v z$. Therefore, equations (6), (7) and (8) hold with $-h v z$ in place of $\frac{\pi}{\ell} \cot \frac{\pi z}{\ell}$, and we have

Lemma 2. For low temperature superconductors, the evolution-drift vector $\boldsymbol{a}(t, x, y, z)$ of movable particles (free electrons) is given by

$$
\left(\sigma^{2} \frac{\partial(R(t, x, y)+S(t, x, y))}{\partial x}, \sigma^{2} \frac{\partial(R(t, x, y)+S(t, x, y))}{\partial y},-\sigma^{2} h v z\right),
$$

where $\sigma^{2}=\frac{\hbar}{m} . \quad$ If

$$
\psi(t, x, y, z)=e^{i(E / \hbar) t} e^{R(x, y)+i\left(S(x, y)-(1 / 2) h v z^{2}\right)},
$$

then on the xy-plane there exists a flow

$$
\boldsymbol{a}(x, y)=\left(\sigma^{2} \frac{\partial(R(x, y)+S(x, y))}{\partial x}, \sigma^{2} \frac{\partial(R(x, y)+S(x, y))}{\partial y}\right)
$$

of movable particles (free electrons). The flow $\boldsymbol{a}(x, y)$ is a permanent electric current, since it is not disturbed by the z-component $-\sigma^{2} h v z$.

Therefore, Lemma 2 implies

Proposition 2. For low temperature superconductors, a permanent electric current $\boldsymbol{a}(x, y)$ in equation (15) occurs at the transition temperature $T_{c}$ given by equation (13) (see equation (5) for the motion of electrons), because the 
z-components of movable particles (free electrons) fall down into the lowest energy state at $T_{c}$, and no longer disturb the electric current $\boldsymbol{a}(x, y)$.

The transition temperature $T_{c}$ in equation (13) contains a parameter $v$, which is a structure constant of a superconductor, for instance, $\mathrm{Hg}$.

\section{Remarks}

Remark 1. Low temperature superconductors such as $\mathrm{Hg}$ have no structure of yellow and white layers as in high temperature superconductors such as $\mathrm{La}_{2-x} \mathrm{Sr}_{x} \mathrm{CuO}_{4}$. A comparison of their transition temperatures

$$
\text { (low) } k_{B} T_{c} \simeq h v \frac{N}{\log N}, \text { and }\left(\text { high) } k_{B} T_{c} \simeq \frac{\hbar^{2}}{2 m} \pi^{2} \frac{1}{\ell^{2}} \frac{2 N}{\log 2 N},\right.
$$

suggest that the lowest energy $\frac{\hbar^{2}}{2 m} \pi^{2} \frac{1}{\ell^{2}}$ of high temperature superconductors is higher than the lowest energy $h v$ of low temperature superconductors. We should notice that both transition temperatures depend also on the number $N$ of particles per unit volume.

Remark 2. It is known (cf., e.g. Fukuyama (1997)) that the occurrence of superconductivity and the transition temperature $T_{c}$ of $\mathrm{La}_{2-x} \mathrm{Sr}_{x} \mathrm{CuO}_{4}$ depend not only on the crystal structure but on the doping $x$ sensitively as illustrated:

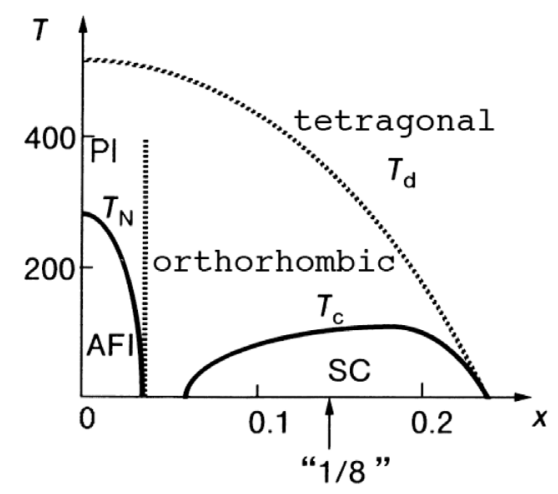

We denote $T_{c}=f(x)$. Then $\frac{1}{k_{B}} \frac{\hbar^{2}}{2 m} \pi^{2} \frac{1}{\ell^{2}} \frac{2 N}{\log 2 N} \simeq f(x)$ in our model. Therefore, the crystal structure constant $\ell^{2}$ and the number $N$ of movable particles (electrons or positive holes) in yellow layers must be functions of $x$ and

$$
f(x) \simeq \frac{1}{k_{B}} \frac{\hbar^{2}}{2 m} \pi^{2} \frac{1}{\ell^{2}(x)} \frac{2 N(x)}{\log 2 N(x)} .
$$

Namely, the function $f(x)$ is a result of a mixture of $\ell^{2}(x)$ and $N(x)$. 
Acknowledgment. I would like to express my thanks to Professor Eiichi Matsuoka of Kobe University for stimulating discussions about the subject treated in this paper.

\section{REFERENCES}

[ 1 ] J. Bardeen, N. CoOper And J. R. SchriefFer, Theory of superconductivity, Phys. Rev. 108 (1957), 1175-1204.

[2] A. Einstein, Quantentheorie des einatomigen idealen Gases, Sittzungsberichte der preussischen Akad. der Wissenschaften Physikalisch-Mathematische Klasse 19 (1924), 261-267.

[3] A. EInstern, Quantentheorie des einatomigen idealen Gases, Zweite Abhandlung, Sitzungsberichte der preussischen Akad, der Wissenschaften Physikalisch-Matematische Klasse 20 (1925), 3-14.

[4] H. Fukuyama, Chapter 2 Superconductors, Theory of matter for graduate students II (M. Date, ed.), Kodansha Scientific, Tokyo, 1997 (in Japanese).

[ 5 ] A. Görlitz, J. M. Vogels, A. E. Leanhardt, C. Raman, T. L. Gustavson, J. R. Abo-Shaeer, A. P. Chikkatur, S. Gupta, S. Inouye, T. Rosenband and W. Ketterle, Realization of Bose-Einstein condensates in lower dimensions, Phys. Rev. Letters 87 (2001), 130402-1/ 130402-4.

[6] M. Nagasawa, Can the Schrödinger equation be a Boltzmann equation?, Evanston, 1989, Diffusion processes and related problems in analysis (M. Pinsky, ed.), Birkhäuser Verlag, Basel, Boston, Berlin, 1990.

[7] M. Nagasawa, Schrödinger equations and diffusion theory, Monographs in mathematics 86, Birkhäuser Verlag, Basel, Boston, Berlin, 1993.

[8] M. Nagasawa, Stochastic processes in quantum physics, Monographs in mathematics 94, Birkhäuser Verlag, Basel, Boston, Berlin, 2000.

[9] M. Nagasawa, On quantum particles, Chaos, Solitons \& Fractals 13 (2002), 1393-1405.

[10] M. Nagasawa, Dynamic theory of stochastic movement of systems, Stochastic economic dynamics (B. J. Jensen and T. Palokangas, eds.), Copenhagen Business School Press, 2007, 133-164.

[11] M. Nagasawa, A mathematical theory for double-slit experiments of Walborn et al, Kodai Math. Journal 35 (2012), 589-612.

[12] M. Nagasawa, New quantum theory with Markov processes, Soeisha, Sanseido Shoten, Tokyo, 2012 (in Japanese).

[13] C. J. Pethick and H. Smith, Bose-Einstein condensation in dilute gases, Cambridge University Press, 2002.

Masao Nagasawa

Institute of Mathematics

UNIVERSITY OF ZÜRICH

WINTERTHURERSTRASSE 190

CH-8057 ZÜRICH

SWITZERLAND 